methasone $50 \mathrm{mg} / \mathrm{d}$ maximum) to treat brain edema which has great influence upon the prognosis of any brain lesion.

Postoperative comatose condition which had been almost fatal has been treated with steroids and most of these cases have survived. $85.0 \%$ of the authors' acute subdural hematoma cases have been rescued since the introduction of corticosteroid therapy for brain edema, whereas only $37.5 \%$ could survive after removal of hematoma before the use of steroids.

In the course of the authors' clinical experience a remarkable difference was found between the steroid group and no-steroid group in the serum transaminase activities. GOT and GPT levels of the steroid-treated patients were kept below the normal levels compared with the non-treated patients after head injury or brain tumor operation.

Histochemical techniques applied to assess the DPN-, TPN-diaphorase of glial cells and Succinic dehydrogenase activity of nerve cells demonstrated that these activities were maintained normal in the steroid group. No increase of glia cell DPN-, TPN-diaphorase activities was observed. Succinic dehydrogenase activity of nerve cells were sufficiently normal.

Biochemical examinations were done to evaluate complications, and failed in finding remarkable complications other than slight retardation of wound healing and very rare exacerbation of digestive tract ulcers. Both of the above mentioned complications were satisfactorily deminished by the use of anabolic steroids.

Mechanism of steroids' effects upon the selective permeability of cell membranes are now studied from molecular-chemical aspects.

\title{
103. Clinical Use of Nucleotides for the Patients of Conscionsness Disorder
}

\author{
Shozo IshII, Haruhiko KikUchi, Hiroshi SaKamoto \\ and Yushi Kondo \\ 1st Surgical Division, Kyoto University
}

The report was made on our experience of nucleotides (CDP-choline, ATP \& caffein) administration on 44 cases who had the disturbance of consciousness.

To those 44 cases of consciousness disturbance due to brain tumor (mainly postoperative) or cerebral vascular lesion, nucleotide was administered on 124 occasions in total.

It was observed to be effective in more than half of these patient $(55 \%)$ and in $33 \%$ of occasion of injection.

In these, unconscious cases due to brain contusion as the result of trauma 
or the operation for brain tumor, responded well to the drug in $78 \%$ of cases. However, in those due to intracranial hemorrhage by various cause, it was effective only in $37 \%$ of cases. When disturbance of consciousness was thought to be apparently due to brain swelling, this treatment was quite effective in all cases.

There was distinct difference in effectiveness of this treatment between the patients above 40 years of age and those below 40 . Effctiveness was observed in $90 \%$ in the group of 0 to 20 years of age, $73 \%$ in those 21 to 40 and only $30 \%$ in those above 40.

The relation between the grade of unconsciousness before the drug administration and the effect of drug revealed that the severer the disturbance of consciousness, the effect was less. Especially, no effect was noted in cases of consciousness disturbance of the dementia form such as apallish state (5 cases).

It was natural that nearly no effect was observed in those who had a massive organic contusion of the brain stem which is known to be closely related to the consciousness.

However, it was thought that the use of nucleotide was not entirely valueless even in those who did not show any improvement in the consciousness level. Because, despite the unimproved state of consciousness, the improvement of cerebral circulatory situation was often evidenced by E.E.G. and R.E.G. findings following the nucleotide administration, thus these will be no doubt that it is acting favourably for the improvement of diseased state to brain.

The combined use of nucleotide and the hypothermia was applied on the cases of hemorrhagic type of brain trauma. A better result was obtained with the survival of 4 out of 6 cases $(66 \%)$ comparing to the result of $28 \%$ when hypothermia only was applied.

\title{
104. Functional Correlation of Brain Cells and Substances Known to Influence the Blood-Brain Barrier (BBB).
}

\author{
Yohji Hara, Jun Tominaga, Toshihiko Kasahara and \\ Tsuneyuki NakazaWA \\ Dept. of Neuropsychiatry, Keio University Medical School
}

To consider the $\mathrm{BBB}$, as a functional entity, is inevitable in any discussion of neuroglial and endothelial functions. The purpose of this report is to extend observations on the behavior of brain cells with specific reference to astrocytes and endothelial cells, under the influence of the substances known to influence the $\mathrm{BBB}$, utilizing such combination of techniques as tissue culture and perfusion chamber with phasecinemicroscopy. 